\title{
Why Urban Mass Demand Responsive Transport?
}

\author{
Jani-Pekka Jokinen, Teemu Sihvola, Esa Hyytiä and Reijo Sulonen \\ Aalto University, School of Science, Finland
}

\begin{abstract}
In this paper, we argue that a large-scale demand responsive system is the missing element from the spectrum of the urban transport modes. To this end, we provide a comprehensive analysis addressing this broad question from several aspects including the customers' perception, various technical aspects, and the related societal aspects. In each case, we are able to give sound arguments why a mass demand responsive transport (DRT) service can improve the current situation considerably. The mass DRT will not make other transport modes obsolete, but can complement them in surprisingly efficient manner leading to considerable internal and external cost savings - a true win-win situation the modern computer and information technology has enabled.
\end{abstract}

\section{INTRODUCTION}

Satisfying a travel demand in a densely populated metropolis is a complicated problem with many aspects. Mass transport is the key component to this end. The main transport modes that are currently in charge of carrying the passengers across the city have been invented, and implemented, decades ago.

In this paper, we consider the applicability of the demand responsive transport (DRT) mode as a potential future concept to satisfy the needs of the urban private car users. We observe that DRT is a superior transport mode simply because i) it is cost effective, ii) it is fundamentally more resilient than taxi and private car, iii) while providing dependable service to passengers, e.g., by freeing them from the burden of timetables. In contrast to the present DRT systems, we propose a mass DRT system capable of producing highquality trips with very short notice times in a cost efficient manner by relying on the modern IT infrastructure.

Obviously, DRT, like any other transport mode, does not satisfy everyone's needs. For example, in busy city centres or in high point-to-point demands, e.g., between an airport and a city centre, it is hard to challenge regular heavy rail or bus lines. Similarly, in sparsely populated rural areas, having too low a travel demand to allow efficient trip combining, there is likely no traffic congestion either, and private cars are a justified solution. The envisioned DRT system complements the current urban transport modes by providing an economic alternative that quickly adapts to a changing travel demand and competes mainly with private cars and taxis in an urban environment. One should make a strong distinction between the traditional forms of DRT (e.g., services tailored for disabled and the elderly), and the large scale open-for-all solutions, whose potential has been initially investigated in [13], and which is also the topic of this paper.

The rest of the paper is organized as follows. First, in Section II we describe the proposed modern era DRT concept, and its abstract simulation model. In Section III, we show that such a DRT system is cost effective, in Section IV we argue that the system is also resilient, while the dependability of the service is considered in Section V. In Section VI, the positive societal impacts are discussed, Section VII summarizes the remaining challenges, and Section VIII concludes the paper.

\section{Preliminaries}

\section{A. Challenges in Modern Urban Transport}

The urban transport problem is, to a great extent, a result of the private car transport [3]. Congestion causes extra costs for travelers and goods transport, loss of time, accidents and stress. Traffic externalities are extensive. Public transport services are provided by the society to alleviate the urban transport problem. However, a large portion of urban travelers do not see these services as real alternatives to private cars [6].

\section{B. Urban Mass Demand Responsive Transport}

DRT provides a shared transport service, which adapts dynamically to demand by routing a fleet of vehicles operating without any fixed routes or timetables. The flexibility of the DRT enables addressing the trade-off between the high quality service of taxi and private car modes, and the efficiency of the public mass transport.

Due to expensive and slow computers, and high cost of operation [11], DRT is nowadays widely adopted only as a transport service for special groups or situations where the demand is too low for other public transport services [3], [4], [1], [10]. These types of systems also require usually heavy subsidization. Recent development in information technology makes it possible to offer DRT service also as an economically viable mass transport service.

To this end, we make some assumptions of the characteristics of an urban mass DRT system. The system could operate in high demand density conditions of an urban metropolis in a competitive position against private car transport. In this scenario, a mass DRT system is assumed to consist of a fully automated scheduling and dispatching system from which customers order trips stop-to-stop typically using handheld devices. Furthermore, no transfers within a trip are needed, i.e., each trip is completed by a single vehicle and the overhead of uncertain transfers is eliminated. No advance reservations are required, and customers can rely on the availability of the service even if the request for a trip is immediate. However, as customers are served stopto-stop, typically trip requests include short pre-order time, 
which guarantee that a customer can arrive in time at the pickup stop. The system can automatically provide more than one option per trip request. Each option includes a service promise to the customer. More concretely, the envisioned service promise consists of three factors as follows. The first factor defines what is the maximum time difference between the target pickup time and the customer defined earliest pickup time (this time interval is labeled as adjustment time). The second factor defines what is the maximum time difference between the actual pickup time and the target pickup time, thus guaranteeing the maximum waiting time. The third factor defines the maximum time difference between the delivery time and the target pickup time (this time interval is labeled as the system time).

\section{Elementary model and example scenario}

To illustrate the service we will utilize an elementary model where trip requests arrive according to a Poisson process to a $10 \mathrm{~km} \times 10 \mathrm{~km}$ rectangular area. In this area, $n$ vehicles operate each capable of transporting at most $c=10$ persons at the same time. Vehicles have a constant speed of $10 \mathrm{~m} / \mathrm{s}$, they move freely in the given area (i.e., there is no road network), and each stop takes at least $30 \mathrm{~s}$ (including deceleration and acceleration). Moreover, there is a regular $250 \mathrm{~m} \times 250 \mathrm{~m}$ stop grid laid over the service area, which is utilized for the DRT service. In all simulation experiments, we have used a 10 hour warm-up period that is followed by a 10 hour simulation period, during which the statistics are collected. In the numerical examples, the 10 hour simulation period is assumed to represent a one day busy period of operations, e.g., a demand density of $1 \mathrm{trip} / \mathrm{km}^{2} /$ hour results in a 1000 trips per day. Some part of this demand is then carried by the DRT system.

The considered DRT system includes the following service promise, which is used as a default unless otherwise stated: The maximum adjustment time is 15 minutes and the maximum waiting time is 5 minutes. The maximum system time is 5 minutes plus 1.5 times the direct ride time of the trip. The factor 1.5 in the maximum system time is labeled as ride time factor (RTF). We have included a service promise also in the taxi system so that the maximum time difference between the order time and pickup time is 15 minutes. Trip requests in the taxi system are immediate, i.e., customers are ready for the pickup at the order time. DRT trip orders, however, include a 5 minute pre-order time, which customers reserve for preparations and walking from trip origin to the pickup stop. Simulations are used for both taxi and DRT modes, while the simplicity of the model allows us to study the private car mode analytically.

A crucifial element is the policy defining the routes of the vehicles in response to trip requests. To this end, we rely on a greedy heuristic policy referred to as minimize-passengertravel-time (MPTT). Basically, MPTT assigns a new trip to a vehicle which can, according to the current information (cf. myopic control), deliver all current passengers, including the new one, with the smallest increase in the sum of the passengers (remaining) travel time. In other words, MPTT does not explicitly take into account the anticipated future requests [9]. For more details on MPTT and other related heuristic policies, we refer to [7], [2], [5], [16]. The optimal control of the vehicle fleet is, in fact, a very complicated problem in general, and one can indeed do better than what this heuristic policy can offer. However, the differences are not qualitative, and as the heuristic MPTT policy already provides on average a relatively good and robust solution, it satisfies the needs of this paper.

Finally, we note that even though the described model is elementary, it captures the essential components of a DRT system: dynamically arriving trip requests, dispatching the vehicles, dynamic route updates, service promises, and the pickup and delivery events.

\section{DRT IS COST EFFECTIVE}

In this section, we study and compare the cost effectiveness of DRT, taxi, and private car. By cost effectiveness we mean that the cost to satisfy a given traffic demand is small when compared with the alternative transport modes. Note that the modes in our comparison cannot compete with mass transport modes such as underground network in situations where the demand is high and the destinations coincide sufficiently. However, the network of underground lines cannot be "reconfigured" based on the changing demand, and building a new line is a massive effort in terms of money and time. In contrast, DRT, taxi and private car can adapt to change in demand "on-the-spot".

We consider cost effectiveness at the various demand levels which enables us to compare the economies of scale of the transport modes. In this section, we consider only internal costs. External costs are considered later in Section VI. Table I presents values for the unit costs. The costs are classified to the fixed costs which are independent of the vehicle kilometers travelled, and to the variable costs which increase linearly with vehicle kilometers. We compare the cost of the alternative transportation modes for a one day period. Therefore, the fixed costs are calculated and presented for a one day. Fixed costs for the taxi and DRT vehicle are defined as a sum of an ownership cost per day and a labour cost of a vehicle driver per day. Whereas a fixed cost of a private car is just ownership costs. We assume that each private car travels 2.9 trips per day. This assumption is based on numbers presented in [15], which describes transportation in the Helsinki metropolitan area. We obtained values for the unit costs from [12]: variable costs are used without any changes from the source, but for the fixed costs we have made some adjustments. The biggest component of the fixed cost after the labour cost is the vehicle depreciation, which is estimated for 24000 kilometers annual usage. Therefore, we adjusted the annual depreciation of taxi and DRT vehicles according to the realized vehicle kilometers, which was on average 105000 kilometers per year for a DRT vehicle and respectively 82000 kilometers for a taxi. We used average bus driver salary $\$ 16.14$ per hour in the United States [from worldsalaries.org] as a labour cost, which is multiplied by 1.3 in order to take into account other related staff expenses. 
TABLE I

COST ITEMS USED IN THE COMPARISON.

\begin{tabular}{|l|l|l|}
\hline Cost item & Definition & Value \$ \\
\hline Fixed cost of private car & Insurance per year, licence \& registration, Deprecia- & 15.33 per day \\
& tion (9320 kilometers/year), financing & \\
Variable cost of private car & Gas \& oil, maintenance, tires & 0.11 per km \\
Fixed cost of taxi & Same as private car + labour cost & $15.33+210.00$ per day \\
Variable cost of taxi & Same as private car & 0.11 per km \\
Fixed cost of DRT & Same as private car + labour cost & $19.92+210.00$ per day \\
Variable cost of DRT & Same as private car & 0.16 per km \\
\hline
\end{tabular}

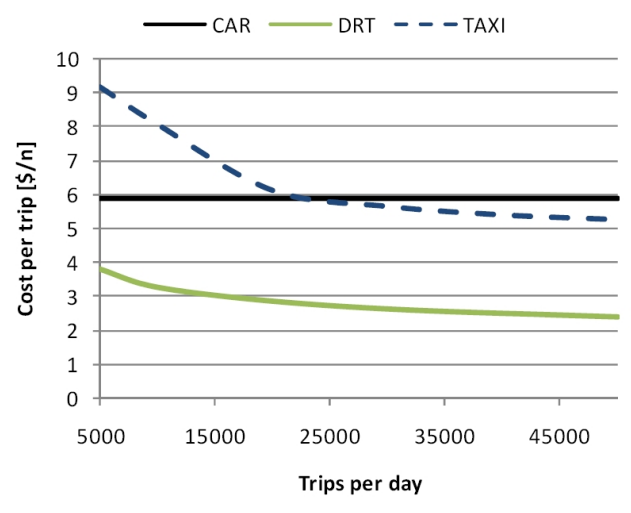

Fig. 1. Estimated average cost per trip as a function of trips per day.

Finally, we added $10 \%$ to the total costs of the DRT and taxi to cover traffic system operation costs. ${ }^{1}$

It turns out that DRT is a very cost effective solution compared to private cars and taxis. Fig. 1 presents cost calculations based on simulation results. The used unit costs are given in Table I. Costs per trip by private car (black line) are invariant at the various demand levels. The costs of taxi and DRT trips decrease as the demand level (demand density) increases, i.e. both transport modes have economies of scale, which is a well-known feature of taxi service in transport literature (see, e.g., [14]).

The mean cost of a trip is clearly lower with DRT than with the other modes at all presented demand levels due to efficient trip combining. ${ }^{2}$ Table II presents essential descriptive numbers from the simulations used in the cost comparisons. The fleet size required to satisfy a given demand is significantly lower in the DRT system than in the other systems at all demand levels. Moreover, the mean occupation of DRT vehicles is naturally also much higher, and it increases along with higher demand levels.

Note that we have not considered yet a travel time cost, which forms a significant part of the total travel costs of the passenger. For example in [12], travel time costs are $16.2 \%$ of all direct travel costs of a private car driver. It is a

\footnotetext{
${ }^{1}$ This is based on our own estimate of the cost of the automated traffic system operation, where a human labour is not needed in trip dispatching and selling, i.e., the only necessary human worker is a vehicle driver

${ }^{2}$ The efficient trip combing requires sufficient demand level. Therefore DRT is not cost effective transport mode compared with the private car in our simulation if the demand level is 1000 trips per day or below
}

challenging task to estimate passenger's value of time spent on traveling by DRT, a travel mode, which differs many ways from the current modes. In Section V, we will present quality of service related features of urban mass DRT, which most likely affect passenger's valuation of time. In this section, we use a straightforward method to estimate the impact of travel time on cost effectiveness. We use Litman's estimate of car driver's travel time value from [12], which is $\$ 0.072$ per minute. ${ }^{3}$ A trip by DRT takes approximately 9 minutes longer than by a private car, ${ }^{4}$ which means a $\$ 0.65$ higher travel time cost. From Fig. 1, we can see that DRT is the most cost effective transport mode despite of the higher travel time cost, because the average cost difference between private car and DRT without time cost is over three dollars. A travel time of a DRT trip includes an adjustment time, i.e., the time between customer defined earliest pickup time and the target pickup time of a trip. As customers can utilize the adjustment time at trip origin for other activities, it is not necessarily correct to include it fully when estimating travel time costs.

\section{DRT IS RESILIENT}

A taxi system is extremely susceptible to an increase in demand. We aim to demonstrate the difference of DRT and taxi with respect to resiliency. By resiliency, we mean the transport system's capability to tolerate an unexpected sudden change in demand without any a priori changes in the operating parameters. Thus, e.g., increasing the number of vehicles, or any other preparation requiring knowledge of the change in demand, is not allowed. Basically, it is important to know how a sudden unexpected increase in demand affects the performance of the system.

In this work, the resiliency is evaluated as follows. Both DRT and taxi fleet sizes are adjusted to correspond to the presumed demand of 30000 trips per day (demand density is $30 \mathrm{trip} / \mathrm{km}^{2} /$ hour). The obtained fleet sizes are shown in Table II: 236 vehicles for DRT and 611 vehicles for taxi. Next we investigate the mean travel time if the demand density is higher than the expected with the same number of vehicles. The example taxi system gives no service promises, e.g., on the pickup time, and the mean travel time simply increases as a function of demand. We assume that the DRT

\footnotetext{
${ }^{3}$ Originally the value was given as per mile. We changed the unit of measurement and adjusted cost value to match travel speed used in simulations.

${ }^{4}$ This comparison ignores the fact that the search time for parking can lengthen the total travel time of a private car significantly.
} 
TABLE II

KEY PERFORMANCE FIGURES.

\begin{tabular}{|ll|rrrrrr|}
\hline Quantity & Transport mode & \multicolumn{5}{|c|}{ Demand level (trip per day = 10hr period) } \\
& & $\mathbf{5 ~ 0 0 0}$ & $\mathbf{1 0}$ 000 & $\mathbf{2 0} \mathbf{0 0 0}$ & $\mathbf{3 0} \mathbf{0 0 0}$ & $\mathbf{4 0} \mathbf{0 0 0}$ & $\mathbf{5 0} 000$ \\
\hline Number of cars/vehicles & Private car & 1724 & 3448 & 6897 & 10345 & 13793 & 17241 \\
& DRT & 57 & 98 & 171 & 236 & 300 & 359 \\
& Taxi & 173 & 302 & 446 & 611 & 776 & 943 \\
\hline Average occupation, & Private car & 1.00 & 1.00 & 1.00 & 1.00 & 1.00 & 1.00 \\
(passenger per vehicle) & DRT & 1.86 & 2.20 & 2.62 & 2.85 & 3.00 & 3.18 \\
& Taxi & 0.87 & 0.89 & 0.90 & 0.90 & 0.90 & 0.90 \\
\hline Mean travel time & Private car & 9.19 & 9.19 & 9.19 & 9.19 & 9.19 & 9.19 \\
(minute) & DRT & 18.05 & 17.77 & 18.11 & 18.10 & 17.90 & 18.05 \\
& Taxi & 11.11 & 10.87 & 10.96 & 11.11 & 11.18 & 11.08 \\
\hline
\end{tabular}

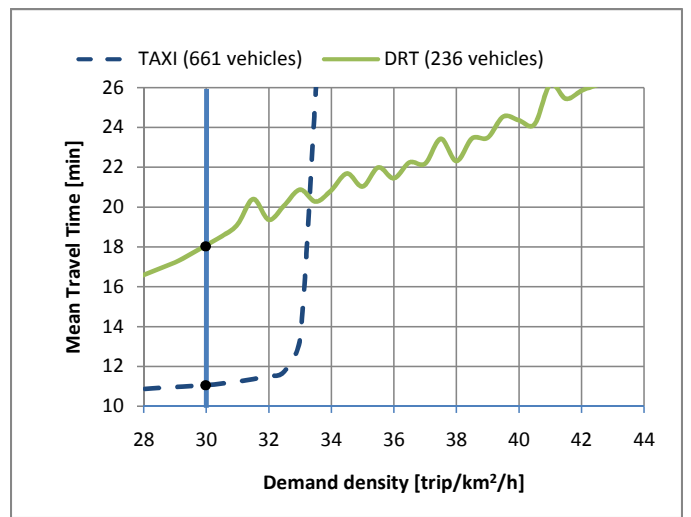

Fig. 2. DRT system is resilient.

operator responds to the unexpected demand increase by relaxing the service promise. Thus, if the default service promise, presented in Section II-C, results in trip rejections, the RTF is increased by 0.1 until no trip request is rejected (with high probability) and the smallest feasible RTF for a given load has been found.

The numerical results are shown in Fig. 2. At the initial demand density, the mean travel time with DRT is about $18 \mathrm{~min}$ and with the taxi service about $11 \mathrm{~min}$. For the reference, a private car achieves a mean travel time of $9 \mathrm{~min}$ and $11 \mathrm{sec}$. If the demand unexpectedly rises, e.g., by about $50 \%$, the DRT system, with appropriately chosen service promises, can still sustain it. In contrast, the example taxi system can tolerate only about $10 \%$ (unexpected) increase in demand.

A good characteristic of a transport system is that the service level is acceptable also during the (unexpected) peak hours. With a taxi system this would mean a seriously overdimensioned system, because it is not possible to introduce flexibility in a taxi system. The same applies, to some extent, also to any transport mode with fixed time tables, e.g., underground and bus lines, where one essentially ends up transporting empty seats during the ordinary hours. In particular, fixed time table transport modes cannot adapt dynamically and utilize the excess capacity in any way. In contrast, apart from being also initially much more cost effective (see the previous section), a DRT system is resilient and in some sense does not need over-dimensioning or reserve capacity. Instead, during the peak hours the mean travel time simply increases modestly. This is very important and favourable property for a transport operator, as bad publicity due to unacceptable quality of service in terms of delays can deteriorate the commercial viability irreversibly. One can also expect that the passengers tolerate somewhat longer travel times during rush hours. After all, DRT offers a significantly better service than the present systems under such circumstances.

\section{DRT ENABLES DEPENDABLE SERVICE PASSENGERS}

The high efficiency of the proposed DRT mode allows one to offer dependable service at the same time. In order to illustrate the quality of service of the DRT compared with the private car transport, we conducted some numerical experiments using the described abstract DRT model. In addition, we envision the pros and cons of the mass DRT service in comparison to the private car and taxi on the basis of the service characteristics presented in the Preliminaries. The key difference between the proposed mass DRT and a traditional taxi system, is that the DRT gives a service promise to the customer, i.e. price and promised quality of service. In a complex system, unpredictable events happen and it is impossible to guarantee that all promises can be kept. However, the system fulfills the requirement for trustworthy and fair service for customers by being dependable in a sense that the best effort is made to keep all promises.

The used service promise, described in Section II-C, enables a reliable and punctual service, which we believe to be an attractive option for a large proportion of the current private car users. Additionally, it is also important that the expected (in contrast to promised) quality of service is sufficiently high, i.e., that the mean travel time components correspond roughly to that of a private car. On the basis of the simulation results, the mean adjustment time can be around $5 \mathrm{~min}$. This time can be possibly rescheduled to other activities. Thus it is less inconvenient than the ride time and, especially, the waiting time. The mean waiting time is around half a minute, meaning that the punctuality of the service is very high. ${ }^{5}$ The mean ride time is about 1.5 times

\footnotetext{
${ }^{5}$ In other words, the delays due to varying traffic conditions will dominate, and other similar transport modes (private car, taxi and bus) are equally susceptible to those.
} 
longer than the direct ride time. Many-to-many DRT service without transfers means that the spatial coverage of the DRT system is on a par with private car transport. Each customer is identified during trip ordering thus diminishing the potential for disorders. This could be relevant especially for current private car users.

Moreover, DRT and taxi are transport services in contrast to the private car transport, where the stress related to driving and parking, and the effort for overhauling the car fall on the car owner and the driver. The nature of real-time operation of mass DRT requires that the system oversees the state of the system and vehicle fleet in traffic, and thus it is possible to offer real time passenger information to the customer in a suitable form. We envision that trips are ordered using modern mobile phones. With well-designed user interfaces, the application is easy to use even for the first time. No understanding of timetables, routes or navigation (if driving private car) is needed. The customer only has to express his/her travel need, and the DRT system provides a service that fulfills the need.

Still, private car includes characteristics which are hard to replace with a taxi or DRT mode. For example, transporting large goods is inconvenient and sometimes impossible. However, instead of using a private car, it is possible to utilize a home delivery service or car sharing. The proposed DRT service is from stop to stop meaning that the walking distance to the stop might be longer than to the parking lot. On the other hand, in urban areas the situation might be also the opposite.

\section{Societal BENEFITS}

The use of private cars creates external costs in the form of congestion, air pollution and noise. A DRT system naturally creates also external costs, but as the total vehicle kilometrage is reduced due to a higher mean occupancy, significant external cost savings can be gained. In this section, we study societal benefits of DRT system in six scenarios, where a certain share of private car users $(0 \%, 10 \%, 20 \%, . .50 \%)$, resulting the total of 100000 trips per day, change their transport mode to the DRT, or in comparison to the taxi, and relinquish private car. We use unit cost values for external cost from [12]. Unit cost values of private car are used for private car and taxi, and the unit cost values of a van with a capacity of 14 passengers is used for a DRT vehicle. The cost values were originally presented separately for urban peak hour, urban of-peak hour and rural travel. We use average external cost values of urban peak and of-peak hour, i.e., we assume that half of the trips are made on peak hours. The used external cost components are congestion, external crash costs, external parking costs, road facilities, land value, traffic services, transport diversity, air pollution, greenhouse gas, noise, resources, barrier effect, land use impacts, water and waste (see detailed descriptions from [12]). The external cost for a private car and taxi is assumed to be $\$ 0.35$ per kilometer and, respectively, $\$ 0.40$ for a DRT vehicle.

As Fig. 3 shows, the external costs decrease almost linearly as the share of DRT increases. In the 0\%-scenario,

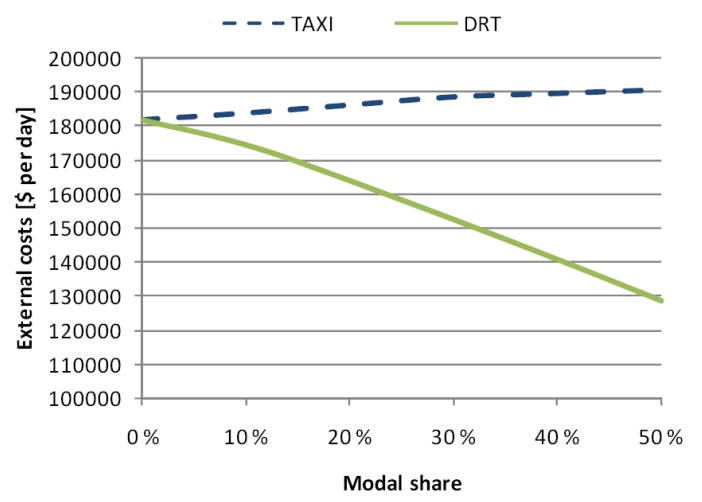

Fig. 3. Estimated external costs (per day) as a function of penetration.

where all trips are made by private cars, the external cost is approximately $\$ 181 \mathrm{k}$ per day. In contrast, in the $50 \%$ scenario, where a half of the private car trips are changed to the DRT trips, the external cost is approximately $\$ 127 \mathrm{k}$ per day, which corresponds to about $30 \%$ reduction in external costs. As can be seen from Fig. 3, in the case of replacing private cars with taxi the external costs increase. This is due to the fact, that with taxis the vehicle often ends up driving empty before the next passenger is picked up.

\section{REMAining Challenges With MASS DRT}

Next we will discuss briefly about the remaining technical and practical challenges.

a) Attitude: As already mentioned, it is hard to break the private car dependency of urban travelers. Moreover, the established actors (transport operators, public sector) are often slow in decision making, and rather trust the old and well-known procedures than experiment with something new. Initial investment is considerable, which also sets a barrier for small size (but agile) businesses.

b) Critical mass: Like any other mass transport, also a fully functioning mass DRT system requires a reasonable large customer base in order to become economically viable. That is, a certain market penetration must be achieved, or the service becomes too expensive for the customers or the society (or both). Naturally, achieving a critical mass is not possible without a competitive pricing scheme.

c) Usability: One strongly related aspect is the usability. One cannot expect the current private car users, or elder people, to be willing to use complicated procedures when ordering a trip. On the other hand, efficiency and fast timescale require an automated system. Thus, a high priority must be given to development of intuitive user-interface through which the trips are ordered and monitored.

d) Technical challenges: The abstract model used in the simulation experiments is a naïve description of the real world. In practice, the environment where a DRT and other transport systems operate is much more hostile due to traffic jams, varying weather conditions, human errors, etc. The fully automated DRT system must be able to cope 
with all types of external uncertainties and changes with a high reliability. To this end, new novel countermeasures that respond in appropriate ways must be developed (note that in this complex system decisions that initially look plausible, can eventually deteriorate the performance totally, cf. congestive collapse [8]). Also the actual implementation, consisting of on-board units, central system(s) and communication between them, poses many technical challenges due to the real-time operation.

\section{DISCUSSION AND SUMMARY}

In this paper, we have proposed and analyzed a novel variant of DRT targeting wider groups in urban areas. More specifically, we have argued that by utilizing the modern computer and information technology, it is possible to develop a new era mass DRT system capable of competing with private cars by offering a very similar service with a fraction of price.

The urban transport problem has many aspects and corresponding challenges. Our model is only an abstract exposition of the service. The used heuristic policy is not optimal and all possibilities of passenger flexibility are not fully utilized. Also, no comprehensive sensitivity analyses for all assumptions were carried out. In any case, the results clearly indicate that the DRT can improve the state of the public transportation significantly. Based on the numerical experiments, we have argued that the proposed DRT system can be i) cost effective, ii) resilient, and iii) dependable service, which has also many positive societal impacts. The cost effectiveness comparison in Section III illustrated that both direct and indirect costs for satisfying daily travel demand can be considerable lower when the proposed DRT system is implemented in an urban environment. In Section VI, we demonstrated that such a system yields also societal benefits in terms of external cost savings, if the system manages to attract private car users to change their transport mode. This gives justification for the society to subsidize DRT, which then enables even more competitive pricing. By resilient, we mean that even under unexpected circumstances, such as a demand peak, the capability of the DRT system to provide service remains at an acceptable level in contrast, e.g., to taxis. By dependable, we mean that the proposed DRT service is capable of offering trips with relatively strict service promises. This is possible due to modern IT infrastructure, which enables real-time communication between vehicles, customers and a central dispatching system. It is essential to understand, that a prerequisite for the societal benefits of an DRT system is the competitiveness against the private car, not against a traditional public transport. On that account, DRT should be positioned as a complement service to the current public transport modes, which improves competitiveness of the whole public transportation sector. For example, in certain situations, a trip combination by underground and DRT could offer a faster and higher quality service than any other relevant transport mode in an urban area.
We believe that the proposed DRT system, utilizing the modern IT infrastructure for real-time communication and coordination, will be the next important step in the field of public transport. In addition to fulfilling the basic travel needs in an efficient manner, there is also room for many related new innovations, e.g., in the field of location-based services. The next step is to develop a real life prototype in Helsinki area. The plan is to start a pilot of the proposed system with a reasonable number of vehicles before the end of 2012 .

\section{ACKNOWLEDGMENTS}

This work was conducted in Metropol project supported by the Finnish Funding Agency for Technology and Innovation, Finnish Ministry of Transport and Communications, Helsinki Region Transport. The authors would like to thank Timo Halko, Lauri Häme, Dr. Aleksi Penttinen, Juha Savolainen and the anonymous reviewers for their useful comments and ideas during the preparation of this paper.

\section{REFERENCES}

[1] P. Bakker. Large scale demand responsive transit systems - a local suburban transport solution for the next millennium. In European Transport Conference, Cambridge, UK, 1999.

[2] L. Bianchi. Notes on dynamic vehicle routing - the state of the art. Technical report, Istituto Dalle Molle Di Studi Sull Intelligenza Artificiale, 2000.

[3] A. Black. Urban mass transportation planning. McGraw-Hill, 1995.

[4] M. Enoch, S. Potter, G. Parkhurst, and M. Smith. Intermode: Innovations in demand responsive transport. Intermode final report, Department for Transport and Greater Manchester Passenger Transport Executive, Manchester, 2004.

[5] M. Gendreau and J. Potvin. Dynamic vehicle routing and dispatching. In T. G. Crainic and G. Laporte, editors, Fleet Management and Logistics, chapter 5, pages 115-125. Springer, June 1998.

[6] R. J. Hjorthol. Mobility in daily life. the car and use of information and communication technology for family logistics. In ERSA conference papers, 2005. European Regional Science Association.

[7] E. Hyytiä, L. Häme, A. Penttinen, and R. Sulonen. Simulation of a large scale dynamic pickup and delivery problem. In $3 \mathrm{rd}$ International ICST Conference on Simulation Tools and Techniques (SIMUTools), 2010.

[8] E. Hyytiä, A. Penttinen, and R. Sulonen. Congestive collapse and its avoidance in a dynamic dial-a-ride system with time windows. In Analytical and Stochastic Modeling Techniques and Applications, pages 397-408, Cardiff, Wales, United Kingdom, June 2010.

[9] S. Ichoua, M. Gendreau, and J. Potvin. Exploiting knowledge about future demands for real-time vehicle dispatching. Transportation Science, 40(2):211-225, May 2006.

[10] T. A. K. Palmer, M. Dessouky. Impact of management practices and advanced technology on demand responsive transit systems. Transportation Research Part A, 38(7):495-509, 2004.

[11] R. E. Lave, R. Teal, and P. Piras. A handbook for acquiring demandresponsive transit software. In Transportation Research Board, Washington, DC, 1996.

[12] A. T. Litman and E. Doherty. Transportation Cost and Benefit Analysis, Techniques, Estimates and Implications. Victoria Transport Policy Institute, second edition, 2009.

[13] T. Sihvola, L. Häme, and R. Sulonen. Passenger-pooling and tripcombining potential of high-density demand-responsive transport. In TRB 89th Annual Meeting, Jan. 2010.

[14] K. A. Small and E. T. Verhoef. The Economics of Urban Transportation. 2007.

[15] H. Strömmer, N. Karasmaa, and R. Valtanen. Helsinki metropolitan area mobility survey 2008. Report, HSL Helsinki Region Transport, 2010.

[16] P. Toth and D. Vigo, editors. The Vehicle Routing Problem. Society for Industrial and Applied Mathematics (SIAM), Philadelphia, USA, 2001. 